\title{
The Future of Dialysis Treatment: Wearable Artificial Kidneys (WAKs)
}

\author{
Rameez Imtiaz, MSc ${ }^{1}$; Tharshika Thangarasa, $\mathrm{BSc}^{1}$ \\ ${ }^{1}$ Faculty of Medicine, University of Ottawa
}

\section{A B STR A C T}

Present day limitations in conventional dialysis therapies have prompted the medical community to explore alternative options in the management of end-stage renal disease (ESRD). The wearable artificial kidney (WAK) is a portable dialysis device, worn around an individual's waist, which aims to provide ESRD patients with sustained hemodialysis. In this paper we will explore the mechanisms by which the WAK operates, as well as the evidence for its efficacy and feasibility in clinical practice.

\section{RÉSUM É}

Les limites actuelles des thérapies de dialyse conventionnelles ont incité la communauté médicale à explorer des options alternatives pour la prise en charge de l'insuffisance rénale chronique. Le rein artificiel portable (WAK, de l'anglais) est un appareil de dialyse portatif, porté autour de la taille d'un individu, qui vise à fournir une hémodialyse continue aux patients atteints d'insuffisance rénale terminale. Dans cet article, nous explorerons les mécanismes de fonctionnement du WAK, ainsi que les preuves de son efficacité et de sa faisabilité en pratique clinique.

\section{INTRODUCTION}

The kidneys play a vital physiological role in maintaining normal health and homeostasis of the human body [1]. They are essential for waste excretion, maintenance of electrolyte and acid-base control, synthesis of vitamin D, and regulation of blood pressure [1]. The consequences of renal insufficiency and eventual failure are devastating, as evidenced by the severely reduced life-span of dialysis patients in comparison to healthy controls [2].

The vast majority of end-stage renal disease (ESRD) patients rely on life-long maintenance dialysis. Dialysis is a process that maintains hematological stability by removing metabolic waste and excess fluid from a patient's bloodstream. In essence, it is designed to replace the hemofiltration functions of the kidneys $[1,3]$. However, this treatment regimen is far from ideal. Current dialysis therapies require patients to have strict limitations on dietary and fluid intake, adherence to a heavy pill burden, and require long periods of limited mobility which, over time, can result in functional limitations [4,5]. Furthermore, accumulating evidence suggests that more frequent dialysis sessions are required to provide better electrolyte control and waste excretion [6]. The limitations of conventional dialysis therapies have prompted the medical community to explore alternative options [4]. Among these recent innovations is the production of a wearable artificial kidney (WAK) by researchers from the University of California, Los Angeles.

The WAK is a miniaturized wearable kidney device which aims to provide ESRD patients with 24-hour dialysis filtration (Figure 1) [4]. In this paper we will explore the mechanisms by which the

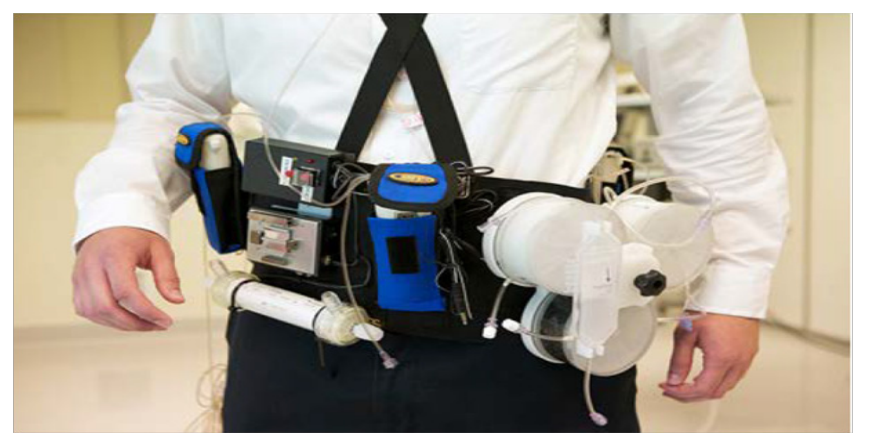

Figure 1: The WAK device worn around the waist [4]

WAK operates, as well as the current evidence supporting its use and its future implications.

\section{Mechanisms facilitating the function of the WAK}

The WAK functions as a portable miniature hemodialysis (HD) machine that can be worn as a belt around the waist. Similar to HD, the WAK is dependent on vascular access to the blood stream. This initiates the passage of blood in a countercurrent flow with the dialysate fluid, which allows for ultrafiltration through diffusion between the two fluids (Figure 2) [7]. Unlike traditional HD which depends on energy consumptive roller pumps to sustain the movement of the blood and dialysate fluid in a coordinated fashion, the WAK relies on small, energy-efficient dual chamber pumps [7]. These pumps are powered by lightweight batteries that prevent irritation of the skin by emitting limited amounts of heat [7].

The WAK also eliminates the requirement for large volumes of 


\section{News \& Letters}

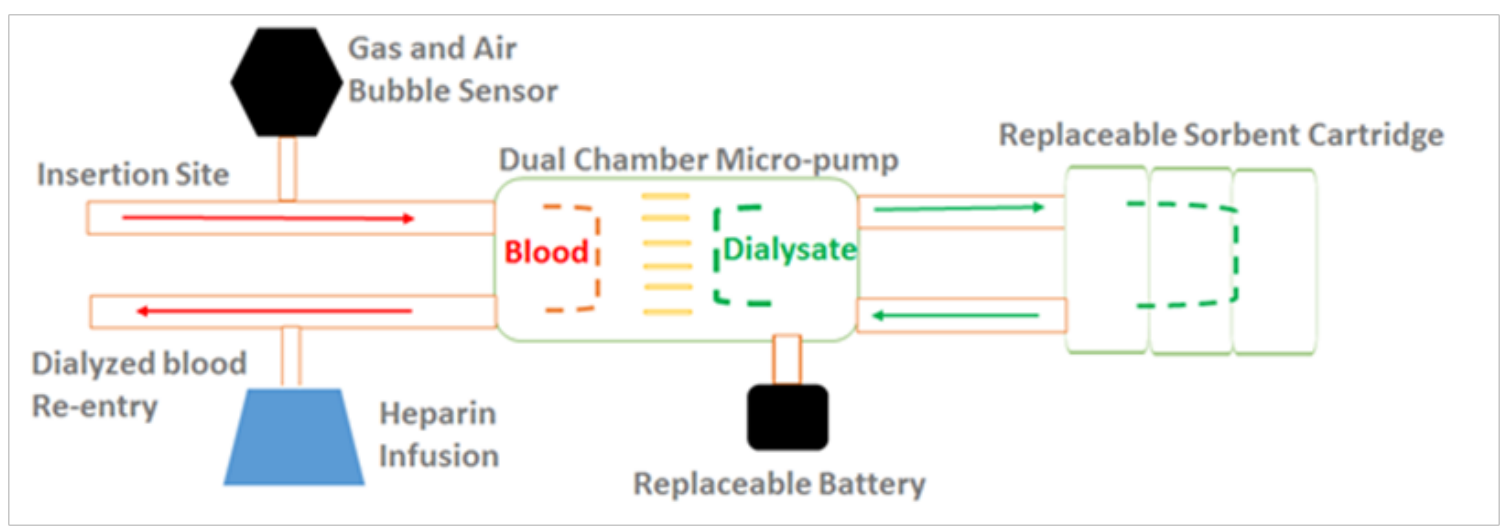

Figure 2: Simplified diagrammatic representation of the components of the WAK

dialysate fluids, which are needed in conventional dialysis to sustain filtration. The WAK is primed with a limited volume of sterile dialysate fluid which is purified by cycling through a series of microporous membranes comprised of sorbent fluids [8]. Sorbent technology consists of various particles that absorb toxins from the dialysate via diffusion across membranes, regenerating the dialysate, and allowing it to filter more blood [7-10]. The sorbent cartridges can then be disposed of at the end of the day to permanently eliminate the dangerous waste metabolites. Fresh sorbent cartridges can be reattached to the WAK to continue the purification process [7].

Furthermore, as an extracorporeal device, the WAK requires continuous access to a patient's blood stream. This can significantly elevate the risk of both blood clot and gas bubble formation [7]. To address these concerns, patients are required to be on heparin infusion, which is delivered directly by the WAK. The WAK is also equipped with a gas bubble sensor that stops blood flow if any bubbles are detected [7].

\section{Clinical Trials of the WAK}

Dr. Victor Gura, a nephrologist turned inventor, pioneered the concept of the WAK and is leading the subsequent clinical trials. Initial animal studies of the WAK, involving pigs as the test subjects, were completed in 2006 [10,11]. These studies demonstrated the WAK to be a safe alternative to conventional dialysis, with no observable side-effects $[10,11]$. This preclinical evidence paved the way for the first pilot study involving the use of the WAK in humans, which was published in 2007 [4]. The study involved eight patients ranging from 26-67 years of age. Patients were on the device for $4-8$ hours (6.4 hours on average) and were allowed to consume food and water as they normally would [4]. The results of the pilot study in humans were promising. Body weight was significantly lower following WAK treatment suggesting that fluids could be successfully removed. Although blood and dialysate flow was lower with the WAK treatment than what is seen in conventional treatment, the WAK allows for extended continuous treatment which could produce clearance rates that exceed that of conventional treatment in the long-term. In addition, the WAK treatment did not cause any adverse changes in patients' blood electrolyte levels and blood $\mathrm{pH}$ remained stable [4]. This pilot study also identified several challenges. Among these, bubbles of carbon dioxide in the dialysate solution created difficulties with blood flow which could potentially attenuate embolism formation. Also, two patients experienced clotting in their access lines suggesting that anticoagulation would be an essential supplement to using the WAK [4].

Following modifications to the original design, another trial was conducted in 2016 with 7 patients (aged 27-73) undergoing WAK treatment over a 24-hour duration [4]. Given that clearance rates of solutes can vary in proportion to blood flow, the flow rate in this study was set lower to minimize fluctuations of blood solute concentrations [4]. The lower flow rate presented certain challenges. Limited clearance of a middle-molecular weight solute 及2-microglobulin was observed, signifying that other middlemolecular weight molecules could also be clearing ineffectively. In addition, a rise in the phosphate levels of some patients was noted. This is of importance because hyperphosphatemia is a marker of poor prognosis in ESRD patients [12]. Regardless, patients reported overall higher levels of treatment satisfaction and no major adverse events were reported.

\section{CONCLUSION}

The current limitations of conventional dialysis have led researchers to explore alternative renal replacement therapies. Among these, the WAK has emerged as the frontrunner. Its performance in clinical trials supports its capabilities of providing successful filtration, while effectively clearing toxins [1]. However, we feel that there are still several issues that need to be addressed. 
These include: determining appropriate flow rates, preventing carbon dioxide bubble formation, defining the most appropriate dosage of anticoagulant, examining the long-term safety and efficacy, and evaluating patient satisfaction after prolonged use. Future clinical trials with a larger number of patients are needed. Finally, researchers must also be wary of issues relating to costs, reimbursements, and other regulations that may accompany the usage of the WAK initially. For these reasons, we feel it is presently premature to consider incorporating the WAK into clinical practice. However, when further clinical trails to better characterize the capabilities of the WAK have been completed, it could prove to be an intriguing option for ESRD patients, improving their quality of life and possibly their prognosis.

\section{REFERENCES}

1. Fissell WH, Shuvo R. The Implantable Artificial Kidney. Semin Dial. 2009;22(6):665-70.

2. Saran R, Li Y, Robinson B, et al. USRDS 2014 annual data report: atlas of endstage renal disease in the United States. Renal Data System. 2014.

3. Abouna GM. Organ shortage crisis: problems and possible solutions. Transplant Proc. 2008;40(1):34-8.

4. Gura V, Matthew RB, Bieber S, et al. A wearable artificial kidney for patients with end-stage renal disease. JCI Insight. 2016;1(8):pii: e86397.

5. Chiu YW, Teitelbaum I, Misra M, De Leon EM, Adzize T, Mehrotra R. Pill burden, adherence, hyperphosphatemia, and quality of life in maintenance dialysis patients. Clin J Am Soc Nephrol. 2009;4(6):1089-96.

6. FHN Trial Group. Chertow GM, Levin NW, et al. In-center hemodialysis six times per week versus three times per week. N Engl J Med. 2010;363(24):2287-300.

7. William FH, Roy S, Davenport A. Achieving more frequent and longer dialysis for the majority: wearable dialysis and implantable artificial kidney devices. Kidney Int. 2014;84(2):256-64.

8. Gura V, Davenport A, Beizai M, Ezon C, Ronco C. $\beta 2$-microglobulin and phosphate clearances using a wearable artificial kidney: a pilot study. Am J Kidney Dis. 2009;54(1):104-11.

9. Agar WMJ. Review: Understanding sorbent dialysis systems. Nephrology. 2010;15(4):406-11.

10. Gura V, Masoud B, Carlos E, Polaschegg H. Continuous renal replacement therapy for end-stage renal disease. The wearable artificial kidney (WAK). Contrib Nephrol. 2005;149:325-33.

11. Gura V, Masoud B, Carlos E, Edmond R. Continuous renal replacement therapy for congestive heart failure: the wearable continuous ultrafiltration system. ASAIO J. 2006;52(1):59-61.

12. Komaba H, Fukagawa $M$. Phosphate-a poison for humans?Kidney Int. 2016;90(4):753-63. 